\title{
Introduction to the Second Adriatic Symposium on Biophysical Approaches in Biomedical Studies
}

\author{
Lawrence Berliner $^{1} \cdot$ Marija Raguz $^{2} \cdot$ Balaraman Kalyanaraman $^{3} \cdot$ Tadeusz Sarna $^{4}$
}

Published online: 26 February 2019

(c) Springer Science+Business Media, LLC, part of Springer Nature 2019

We are pleased and honored to present this special issue for CBBI on recent advances in selected biophysical methods to study biological systems, oxidative phenomena involved in the pathology of human diseases, free radical metabolism and bioenergetic changes in cardiovascular diseases and cancer, and advances in drug, active surfaces and biosensor development. The papers herein resulted from the most recent 2017 Second Adriatic Symposium on Biophysical Approaches in Biomedical Studies held in Split, Croatia, that encompasses work from outstanding researchers in the field.

This is the second in a series of Symposia to be held in Split to share the latest technological developments in various biomedical applications from Croatian and international experts, stimulate innovative research, train young scientists, postdoctoral fellows, graduate students, and enable future scientific collaborations among meeting participants. Invited speakers have been drawn from the leaders in interdisciplinary fields. Scientists from academia, industry and government laboratories from all over the world participated, coming from fields of biophysics, biology, chemistry, biomedicine, and others. Our participants perform are researchers using analytical and biophysical techniques including EPR, NMR, fluorescence and other spectroscopies, microscopy, and nanoscale technologies.

\footnotetext{
Lawrence Berliner

berliner@du.edu

1 Cell Biochemistry and Biophysics, Denver, CO, USA

2 University of Split, Split, Croatia

3 Medical College of Wisconsin, Milwaukee, WI, USA

4 Jagiellonian University, Kraków, Poland
}

\section{Short History of Adriatic Symposium on Biophysical Approaches in Biomedical Studies Organized in Split}

The idea to invite word-leading investigators in the field of biophysics to the University of Split first came from Prof. Balaraman Kalyanaraman in 2012. Adriatic Symposia on Biophysical Approaches in Biomedical Studies has been jointly organized in 2014 and 2017 by scientifically recognized researchers from the University of Split School of Medicine, Split, Croatia, Department of Biophysics at the Medical College of Wisconsin, Milwaukee, USA, and the Department of Biophysics at Jagiellonian University, Krakow, Poland at the Mediterranean Institute for Life Sciences, in Split. Both times symposia were organized by Assoc. Prof. Marija Raguz (Split), Prof. Balaraman Kalyanaraman (Milwaukee), and Prof. Tadeusz Sarna (Krakow). The symposium incorporated a traditional format of plenary lectures, which highlights major advances in each of the major areas that were followed by short lectures from selected abstracts as well as poster presentations.

\section{Layout of the Special Issue}

This is the first Symposium honored with a special issue in CBBI. Papers are related to the following topic areas: advances in magnetic resonance techniques, imaging biomarkers in living systems, biophysical photo-techniques, redox bioenergetics and metabolism diseases, lateral organization and dynamics of biological membranes, lipid and protein modifications: implications in diseases, molecular simulations and mathematical modeling, and free radical detection in vivo and in vitro.

The editors hope that you enjoy this issue and learn about the impressive progress that has occurred to date. 